\title{
Optimized Ge nanowire arrays on Si by modified surfactant mediated epitaxy
}

\author{
K. Romanyuk, ${ }^{1}$ J. Mysliveček, ${ }^{1,2}$ V. Cherepanov,${ }^{1}$ T. Sekiguchi, ${ }^{3}$ S. Yoshida, ${ }^{3}$ K. M. Itoh, ${ }^{3}$ and B. Voigtländer ${ }^{1, *}$ \\ ${ }^{1}$ Institute of Bio and Nanosystems (IBN 3), and CNI (Center of Nanoelectronic Systems for Information Technology), \\ Research Centre Jülich, 52425 Jülich, Germany \\ ${ }^{2}$ Department of Surface and Plasma Physics, Faculty of Mathematics and Physics, Charles University in Prague, \\ V Holešovičkách 2, 18000 Praha 8, Czech Republic \\ ${ }^{3}$ Department of Applied Physics and Physico-Informatics, Keio University, 3-14-1, Hiyoshi, Kohoku-ku, Yokohama, 223-8522, Japan
}

(Received 23 April 2007; published 29 June 2007)

\begin{abstract}
We demonstrate the formation of Ge nanowire arrays on highly ordered kink-free Si stepped surfaces. The nanowires are grown using Bi surfactant mediated epitaxy. The nanowires are single crystalline and feature minimal kink densities, allowing them to span lengths larger than $1 \mu \mathrm{m}$ at a width of $\approx 4 \mathrm{~nm}$. To achieve desired growth conditions for the formation of such nanowire arrays, we explore a full parameter space of surfactant mediated epitaxy. We show that controlling the surfactant coverage in the surface and/or at step edges modifies the growth properties of surface steps in a decisive way.
\end{abstract}

DOI: $10.1103 /$ PhysRevB.75.241309

PACS number(s): 61.46.-w, 81.16.Dn, 81.15.Hi, 68.37.Ef

In order to fabricate ever-smaller nanoscale device structures, there is an enormous interest in finding ways to build devices from the bottom up rather than fabricate from the top down. Using the bottom-up approach, the size of the structures is not limited by lithography; however, the uniformity and the ability to position the nanostructures are still challenges. Specifically, nanowires are desirable as nanoscale interconnects. ${ }^{1}$ One of the concepts followed in the bottom-up formation of nanowire arrays is to create a highly ordered atomic-step template on a vicinal single-crystal surface and to form the wires along the step edges, decorating the step edges with a selected material. ${ }^{2-4}$ A suitable template is the vicinal surface of a $\operatorname{Si}(111)$ single crystal, since the structure of the step train on this surface can be controlled to a large extent. ${ }^{5-7}$ Steps on $\mathrm{Si}(111)$ vicinal surfaces have been decorated by metals, ${ }^{3,8}$ semiconductors, ${ }^{4,9}$ and organic molecules. ${ }^{10}$ The challenge is to improve the homogeneity of the nanowire array and the crystallinity and the aspect ratio of the wires.

Recently, we made two contributions to these efforts. First, we developed a method to grow high-aspect-ratio single-crystalline $\mathrm{Ge}$ nanowires at $\mathrm{Si}(111)$ step edges. ${ }^{4}$ We used surfactant mediated epitaxy ${ }^{9}$ where the surfactant $(\mathrm{Bi})$ always floats at the growth front, covering both the Si surface and the Ge nanowires. The surfactant prevented mutual Ge-Si intermixing and mediated a chemical contrast between $\mathrm{Si}$ and Ge, allowing observation of the lateral Ge-Si nanostructures in a scanning tunneling microscope (STM). Second, we developed a method to obtain an improved ordering of the step train on the $\mathrm{Si}(111)-7 \times 7$ surface by precise mechanical polishing of $\mathrm{Si}$ wafers and subsequent controlled annealing in vacuum. ${ }^{7}$ We obtain surface steps that are kinkfree, atomically straight, and largely equidistant at scales of $1 \times 1 \mu \mathrm{m}^{2}$ [Figs. 1(a) and 1(b)]. ${ }^{7}$

In this work we show how it is possible to create a highly ordered array of Ge nanowires on the Bi-covered $\mathrm{Si}(111)$ surface [Figs. 1(c)-1(f)]. A straightforward combination of the above two techniques ${ }^{4,7}$ does not yield the desired result. The important point is to conserve the long-range order of the initial Si template, which is usually lost during the nano- wire preparation process using standard surfactant mediated epitaxy (as in Ref. 4). We introduce the concept of modified surfactant mediated epitaxy: in critical stages of the growth we temporarily reduce the standard 1 monolayer (ML; $1 \mathrm{ML}=7 \times 10^{14}$ atoms $\mathrm{cm}^{-2}$ ) Bi surface coverage $\mathrm{B}^{4,11,12}$ to influence the growth and equilibrium properties of the surface steps in a desired way. Proper adjustment of the Bi surfactant coverage on terraces or at step edges allows one to fabricate Ge nanowire arrays with a homogeneous width in the onedigit nanometer range having an equidistant spacing and lengths over $1 \mu \mathrm{m}$.

Our experiments were performed in an ultrahigh-vacuum chamber with a base pressure of $5 \times 10^{-11}$ mbar. Bi was evaporated from a Knudsen cell and Ge was evaporated from a tantalum crucible heated by an $e$ beam. Si substrates were heated by passing dc current. The resulting surface structures were observed by STM after quenching the samples to room temperature. We can divide the procedure of preparation of the Ge nanowire array into three stages: (i) preparation of the highly ordered $\mathrm{Si}(111)$ template, (ii) termination of the template by $1 \mathrm{ML} \mathrm{Bi}$, and (iii) growth of Ge nanowires on the Bi-terminated template.

(i) The highly ordered $\mathrm{Si}(111)-7 \times 7$ template was prepared as in Ref. 7. We polished a $\mathrm{Si}(111)$ wafer to $1^{\circ}$ miscut $\left(\theta_{m}\right)$ toward the $\left[\begin{array}{ll}\overline{1} & \overline{1} 2\end{array}\right]$ direction with an intentional azimuthal misorientation $\left(\phi_{m}=4^{\circ}\right)$ to orient all kinks at the surface steps in the same direction. The samples were cleaned in vacuum with flash heating to $1300{ }^{\circ} \mathrm{C}$ and rapidly quenched. Afterward, the samples were annealed at $800-830{ }^{\circ} \mathrm{C}$ for about $10 \mathrm{~h}$ with dc current flowing parallel to the steps in the "kink-up" direction" to extend the atomically straight step edges by surface electromigration of $\mathrm{Si}$. The resulting surface step structure is shown in Fig. 1(a). The kink-up direction was used for dc sample heating in all subsequent preparation steps.

(ii) The standard Bi surfactant mediated epitaxy $y^{4,13}$ on the Si(111) $-7 \times 7$ surface starts by terminating the surface with 1 ML of Bi which forms a $\sqrt{3} \times \sqrt{3}$ surface structure. ${ }^{14-16}$ This is associated with a significant surface mass transport of $\mathrm{Si}$, because $0.24 \mathrm{ML}$ of $\mathrm{Si}$ atoms are released as a result of the 

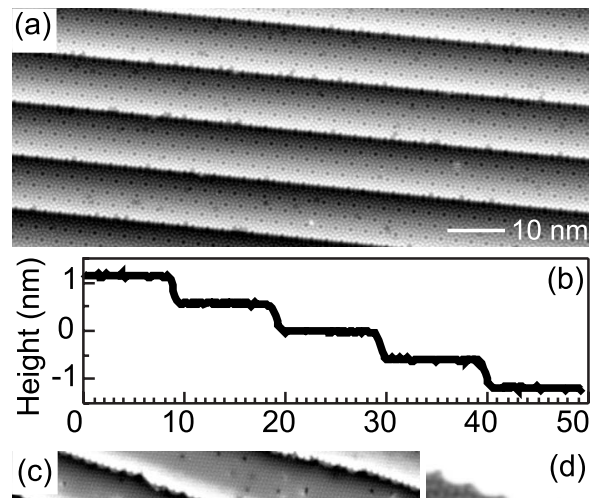

(d)
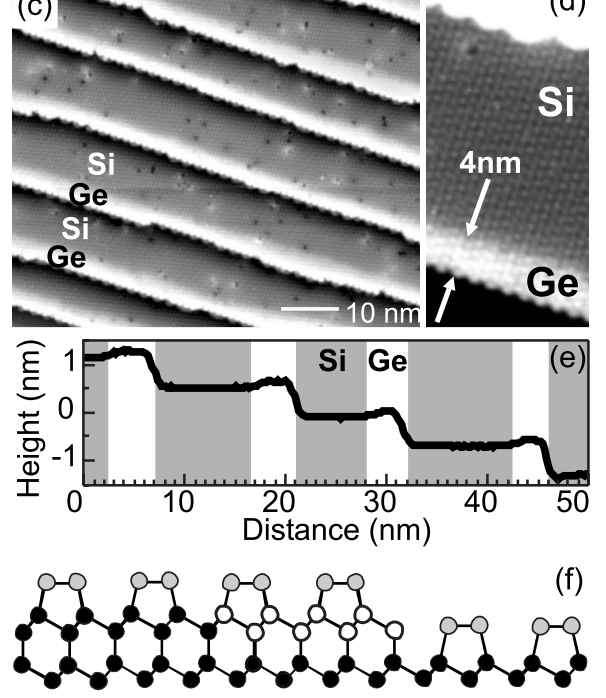

OBi capping OGe bilayer $\bullet$ Si bulk

FIG. 1. (a) Highly ordered $\mathrm{Si}(111)-7 \times 7$ template. (b) Corresponding height profile. (c),(d) Ge nanowires grown on this template by modified surfactant mediated epitaxy. The long-range order of the template remains conserved. The Bi surfactant changes the surface reconstruction to $\sqrt{3} \times \sqrt{3}$ (d). (e) Apparent height difference measured by STM between Si and Ge areas. The nanowires are Ge bilayers attached to the step edges under the Bi capping (f). They measure $\sim 4 \mathrm{~nm}(\sim 20$ atoms $)$ in width and $0.3 \mathrm{~nm}$ in height.

lifting of the $7 \times 7$ surface reconstruction. ${ }^{17}$ Performing the termination in a standard way at substrate temperature $T_{s}$ $=500{ }^{\circ} \mathrm{C}$ and terminating with a Bi flux $F_{\mathrm{Bi}}=0.7 \mathrm{ML} / \mathrm{min}$ for $\approx 10$ min results in a need to incorporate $0.24 \mathrm{ML} \mathrm{Si}$ at the surface step edges in a relatively short time. In Fig. 2(a) we show the result of such standard $\mathrm{Bi}$ termination performed on a highly ordered Si template [Figs. 1(a) and 1(b)]. The steps become wavy on a short length scale and the step ordering is lost [Fig. 2(a)].

We solve this problem by a careful control of the Bi coverage at the terraces during $\mathrm{Bi}$ termination. We use the fact that $\mathrm{Bi}$ readily desorbs from the $\mathrm{Si}(111)$ surface at elevated temperatures. The rate of desorption is $F_{d e s}=0.5 \mathrm{ML} / \mathrm{min}$ at $T_{s}=550{ }^{\circ} \mathrm{C} .{ }^{18,19}$ Thus, for a constant Bi flux we can control the $\mathrm{Bi}$ coverage in a steady-state regime by adjusting the $T_{s}$.

In the optimized $\mathrm{Bi}$ termination procedure, we heat the substrate to $T_{s}=700{ }^{\circ} \mathrm{C}$ and start to deposit $\mathrm{Bi}$ at $F_{\mathrm{Bi}}$ $=3 \mathrm{ML} / \mathrm{min}$. At this high temperature the $\mathrm{Bi}$ coverage is virtually zero. Afterward, we reduce $T_{s}$ to $650{ }^{\circ} \mathrm{C}$ over $10 \mathrm{~min}$. This leads to a slow increase of the $\mathrm{Bi}$ coverage,
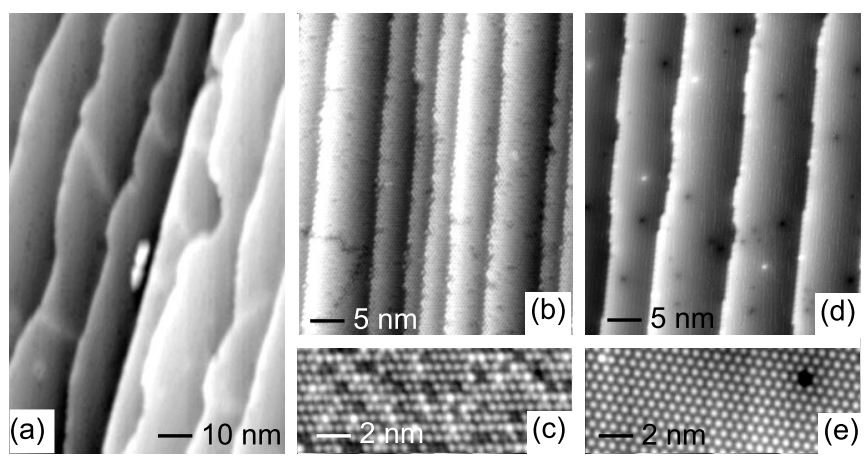

FIG. 2. Bi termination of the highly ordered $\mathrm{Si}(111)-7 \times 7$ template [Fig. 1(a)]. (a) Standard surfactant mediated epitaxy. The step structure of the template has been destroyed. (b)-(e) Modified surfactant mediated epitaxy. (b) Surface after slow increase of Bi coverage from 0 to $1 / 3 \mathrm{ML}$. The $1 / 3 \mathrm{ML}$ Bi coverage is indicated by an inhomogeneous $\sqrt{3} \times \sqrt{3}$ structure (c). The modified surfactant mediated epitaxy preserves the original straight step arrangement. (d) Surface after completing the Bi coverage to $1 \mathrm{ML}$. The $1 \mathrm{ML} B \mathrm{Bi}$ coverage is indicated by a homogeneous $\sqrt{3} \times \sqrt{3}$ structure (e).

allowing a gradual Si mass transport during the lifting of the Si(111)-7 $\times 7$ reconstruction. On Fig. 2(b) we show the morphology of the surface after this preparation step. The surface steps remained straight, indicating that the released $\mathrm{Si}$ atoms had enough time to evenly distribute along the step edges. The detailed view of the surface reveals an inhomogeneous $\sqrt{3} \times \sqrt{3}$ surface structure [Fig. 2(c)], indicative of a 1/3 ML Bi coverage. ${ }^{15}$ The protrusions observed in the STM image of the 1/3 ML Bi structure correspond to Bi monomers on the $\mathrm{Si}(111)$ substrate.

Before Ge deposition, $\mathrm{Bi}$ coverage has to be increased to $1 \mathrm{ML}$ because only $1 \mathrm{ML} \mathrm{Bi}$ termination prevents $\mathrm{Ge}-\mathrm{Si}$ intermixing on the terraces during the growth of the $\mathrm{Ge}$ nanowires. ${ }^{4}$ Thus, we deposit $\mathrm{Bi}$ at $F_{\mathrm{Bi}}=3 \mathrm{ML} / \mathrm{min}$ on the $1 / 3 \mathrm{ML} \mathrm{Bi}$ terminated surface at $T_{s}=500{ }^{\circ} \mathrm{C}$ for $1 \mathrm{~min}$. This causes a slight increase of the kink density. However, the step structure of the original highly ordered step train remains largely conserved, as we can see in Fig. 2(d). The surface reveals a homogeneous $\sqrt{3} \times \sqrt{3}$ surface structure indicative of $1 \mathrm{ML}$ Bi coverage [Fig. 2(e)]. ${ }^{15}$ The protrusions observed in the STM image of the $1 \mathrm{ML} \mathrm{Bi}$ structure correspond to $\mathrm{Bi}$ trimers on the $\mathrm{Si}(111)$ substrate. No $\mathrm{Si}$ mass transport is involved in this part of the process. The underlying $1 \times 1 \mathrm{Si}(111)$ structure is preserved, only the Bi monomers become Bi trimers.

(iii) In the last preparation stage we grow the Ge nanowires along the step edges of the $1 \mathrm{ML}$ Bi terminated template [Figs. 2(d) and 2(e)] using surfactant mediated epitaxy. ${ }^{4,11,19}$ Ge grows in step flow growth mode and a thin stripe of Ge attaches to the step edge. In standard surfactant mediated epitaxy, materials are deposited under a constant supply of the surfactant to maintain the (saturated) $1 \mathrm{ML}$ surfactant coverage. ${ }^{13,16}$ However, performing the Ge deposition in the standard way at $F_{\mathrm{Ge}}=0.08 \mathrm{ML} / \mathrm{min}, T_{s}$ $=450{ }^{\circ} \mathrm{C}$, and Bi flux $F_{\mathrm{Bi}}=3 \mathrm{ML} / \mathrm{min}$ does not yield straight $\mathrm{Ge}$ nanowires of homogenous width. In Fig. 3(a) we can observe that Ge nanowires grown by standard surfactant mediated epitaxy develop step edges in the $\langle 1 \overline{1} 0\rangle$, directions 

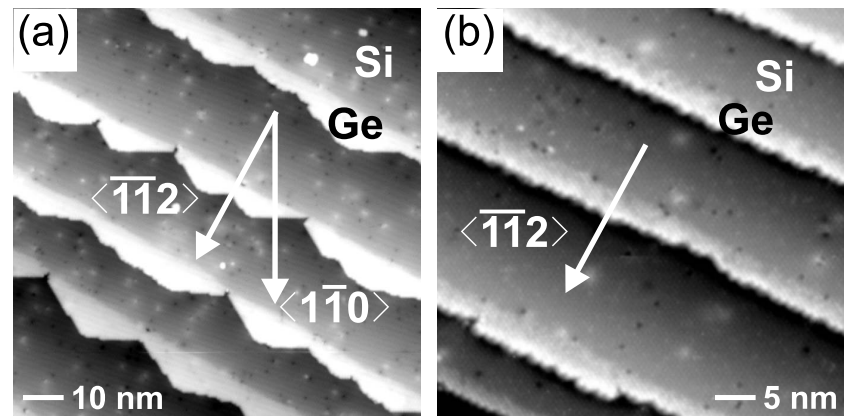

FIG. 3. Deposition of Ge on the ordered Bi-terminated surface [Fig. 2(d)]. (a) Standard surfactant mediated epitaxy. Ge and Bi are deposited simultaneously. The Ge step edges develop facets in the $\langle 1 \overline{1} 0\rangle$ directions that are $30^{\circ}$ off the original $\langle\overline{1} \overline{1} 2\rangle$ step direction. (b) Modified surfactant mediated epitaxy. Ge is deposited without Bi flux. The Ge step edges retain the original $\langle\overline{1} \overline{1} 2\rangle$ step direction.

which are $30^{\circ}$ off the step direction of the template.

To obtain the desired regular growth of Ge nanowire arrays we again consider reducing the $\mathrm{Bi}$ surfactant coverage. To minimize the Ge-Si intermixing, we switch off the Bi flux only during $\mathrm{Ge}$ evaporation and deposit $\mathrm{Ge}$ at $T_{s}=400{ }^{\circ} \mathrm{C}$, $F_{\mathrm{Ge}}=0.02 \mathrm{ML} / \mathrm{min}$ for $10 \mathrm{~min}$. Afterward, we cool the sample rapidly to room temperature. We show the result of this preparation step in Figs. 3(b), 1(c), and 1(d). We obtain the optimized growth of $\mathrm{Ge}$ at the template step edges, resulting in single-crystalline, high-aspect-ratio, low-kinkdensity Ge nanowires with width of about $4 \mathrm{~nm}$.

The success of the last preparation step shows that switching off the $\mathrm{Bi}$ flux at $T_{s}=400{ }^{\circ} \mathrm{C}$ is sufficient to change the Ge growth scenario. We cannot observe any significant reduction of $\mathrm{B}$ i concentration on the terraces after this growth step. The $\sqrt{3} \times \sqrt{3}$ surface structure is homogeneous, indicating the saturation $1 \mathrm{ML} \mathrm{Bi}$ coverage [Fig. 1(d)]. ${ }^{15}$ Therefore, we attribute the change of growth scenario to a reduction of Bi coverage at the step edges.

The optimized growth of a Ge nanowire array on a highly ordered $\mathrm{Si}(111)$ template has been achieved by modified surfactant mediated epitaxy where the surfactant coverage on the terraces [stage (ii)] or at step edges [stage (iii)] is lowered below the saturation coverage. In the following we argue that the observed modifications of the growth scenario with changing $\mathrm{Bi}$ coverage are due to the modified equilibrium properties of step edges in combination with kinetic limitations during growth.

The $\langle\overline{1} \overline{1} 2\rangle$ step direction of our highly ordered templates is the equilibrium step direction of the $\mathrm{Si}(111)-7 \times 7$ surface ${ }^{5}$ Fig. 4(a). We performed an experiment that shows that, upon a complete $1 \mathrm{ML} \mathrm{Bi}$ termination and after prolonged annealing of the surface under $\mathrm{Bi}$ flux, the preferred step direction on the $\mathrm{Si}(111)-\sqrt{3} \times \sqrt{3}$ - $\mathrm{Bi}$ surface changes to $\langle 1 \overline{1} 0\rangle$, i.e., it rotates by $30^{\circ}$. In Fig. 4(b) we can see that at the $\langle 1 \overline{1} 0\rangle$-oriented equilibrium steps the trimers of the $\sqrt{3} \times \sqrt{3}$ surface reconstruction are most densely packed. The fact that the $1 \mathrm{ML} \mathrm{Bi}$ termination of the $\mathrm{Si}(111)$ surface changes the equilibrium step direction explains the general difficulty in growing Ge nanowires on $\mathrm{Si}(111)-7 \times 7$ stepped templates.
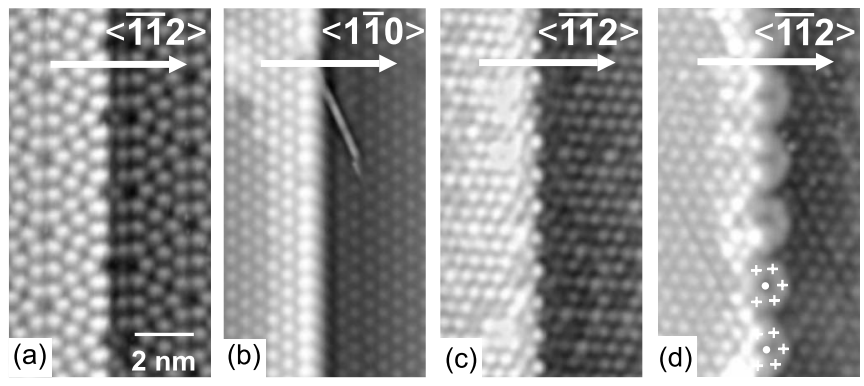

FIG. 4. Changing the $\mathrm{Bi}$ content in the surface changes the structure of the step edges and their growth and equilibrium properties. The equilibrium step directions are (a) $\langle\overline{1} \overline{1} 2\rangle$ on the clean $\mathrm{Si}(111)-7 \times 7$ surface, (b) $\langle 1 \overline{1} 0\rangle$ on the $\mathrm{Si}(111)-\sqrt{3} \times \sqrt{3}-\mathrm{Bi}$ with 1 ML Bi, (c) $\langle\overline{1} \overline{1} 2\rangle$ on the $\mathrm{Si}(111)-\sqrt{3} \times \sqrt{3}-\mathrm{Bi}$ surface with $1 / 3 \mathrm{ML}$ $\mathrm{Bi}$, and $(\mathrm{d})\langle\overline{1} \overline{1} 2\rangle$ on the Ge nanowires grown by modified surfactant mediated epitaxy. In (d), the crosses and dots indicate the positions of the adatoms and corner holes of a $3 \times 3$ surface reconstruction.

When the $\operatorname{Si}(111)-7 \times 7$ ordered template is terminated by 1/3 ML Bi [as in stage (ii), Figs. 2(b) and 2(c)], the preferred low-energy step direction remains $\langle\overline{1} \overline{1} 2\rangle$. Our experiment shows that this step direction is stable in spite of the relatively open structure of the step edge that can be seen in Fig. 4(c). This indicates that the equilibrium step direction is strongly influenced by the actual amount of $\mathrm{Bi}$ in the surface, and, in turn, at the step edges.

Given the above arguments, it could be expected that, after increasing the $1 / 3$ ML Bi coverage of the highly ordered stepped template to $1 \mathrm{ML}$ in stage (ii), the step direction changes. However, this does not happen due to a kinetic limitation. The expected $\langle 1 \overline{1} 0\rangle$ facets do not form because the straight step edges of the template with $1 / 3 \mathrm{ML} B i$ do not emit enough $\mathrm{Si}$ adatoms at the temperature used in this preparation step $\left(T_{s}=500{ }^{\circ} \mathrm{C}\right)$ to allow surface equilibration within the given time $(1 \mathrm{~min})$. The step orientation remains $\langle\overline{1} \overline{1} 2\rangle$, Fig. 2(d).

Finally, in stage (iii), Ge is deposited on the $1 \mathrm{ML}$ Biterminated template. In this case the Ge adatoms do not have to detach from existing step edges but are supplied from outside. This, together with the lower effective activation energy for the Ge surface diffusion on the Bi-terminated $\mathrm{Si}(111)$ surface $^{20}$ leads to the formation of $\langle 1 \overline{1} 0\rangle$ equilibrium facets that destroy the ordered step train [Fig. 3(a)]. However, only a slight decrease of the Bi content on the surface during the Ge deposition is sufficient to preserve the original $\langle\overline{1} \overline{1} 2\rangle$ orientation of the steps [Fig. 3(b)]. In Fig. 4(d) we show that the microscopic structure of the $\langle\overline{1} \overline{1} 2\rangle$ step edge of the Ge nanowire formed during growth without Bi codeposition considerably differs from the structure of the equilibrium steps on the $1 \mathrm{ML}$ and 1/3 ML Bi-terminated surfaces [Figs. 4(b) and 4(c)]. This observation confirms that during Ge growth the surfactant is removed from the step edges, which strongly influences the growth properties of the steps.

We can directly observe the Bi depletion at the step edge in Fig. 4(d). The step edge has a periodic ringlike structure 
with a period corresponding to double the period of the $\mathrm{Si}(111)-3 \times 3$ surface reconstruction. $\mathrm{Si}(111)-3 \times 3$ is commensurate with $\sqrt{3} \times \sqrt{3}$. It is a metastable structure ${ }^{21}$ which was observed on disordered $\mathrm{Si}(111)$ surfaces. ${ }^{22}$ We can identify the corner holes [dots in Fig. 4(d)] and the adatoms [crosses in Fig. 4(d)] of the $3 \times 3$ structure located at the step edge. The adatoms have $\sim 2 \AA$ lower apparent height than the $\mathrm{Bi}$ trimers on the upper terrace. This height difference corresponds to the height difference we measure on samples, where larger areas of $1 \mathrm{ML}$ terminated $\mathrm{Si}(111)-\sqrt{3} \times \sqrt{3}-\mathrm{Bi}$ and clean $\mathrm{Si}(111)-7 \times 7$ coexist. This confirms the assignment of the ringlike arranged adatoms as $\mathrm{Si}$ or $\mathrm{Ge}$ without $\mathrm{Bi}$ termination.

To conclude, we introduce a technique of modified surfactant mediated epitaxy, where we reduce the surfactant concentration on the surface to influence the growth and equilibrium properties of the surface steps in a desired way. We show that this modified surfactant mediated epitaxy is relevant to nanotechnology since it allows fabricating arrays of long equidistant epitaxial Ge nanowires with a width in the one-digit nanometer range. In future these nanowire arrays could serve as templates for selective attachment of molecules, decoration with metals, or attachment of clusters and other nanoscale building blocks such as fullerenes. The study of charge transport through such very small nanowires will be another challenge. Our present work focuses on one specific material combination. However, the use of surfactant coverage as a variable parameter opens up an additional dimension in the growth parameter space that can be explored in bottom-up nanofabrication studies in general.

We gratefully acknowledge the technical support from Helmut Stollwerk and Peter Coenen. This work was supported in part by Grant-in-Aid for Scientific Research by MEXT, Specially Promoted Research \#18001002 and in part by Special Coordination Funds for Promoting Science and Technology. K.R. acknowledges the support of the European Union within the NANOCAGE Marie Curie early stage training network. *b.voigtlaender@fz-juelich.de

${ }^{1}$ E. Tutuc, J. Appenzeller, M. C. Reuter, and S. Guha, Nano Lett. 6, 2070 (2006).

${ }^{2}$ T. Jung, R. Schlittler, J. K. Gimzewski, and F. J. Himpsel, Appl. Phys. A: Mater. Sci. Process. 61, 467 (1995); F. J. Himpsel, T. Jung, and J. E. Ortega, Surf. Rev. Lett. 4, 371 (1997); F. J. Himpsel, T. Jung, A. Kirakosian, J.-L. Lin, D. Y. Petrovykh, H. Rauscher, and J. Viernow, MRS Bull. 24, 20 (1999).

${ }^{3}$ J. Viernow, D. Y. Petrovykh, F. K. Men, J.-L. Lin, and F. J. Himpsel, Appl. Phys. Lett. 74, 2125 (1999).

${ }^{4}$ M. Kawamura, N. Paul, V. Cherepanov, and B. Voigtländer, Phys. Rev. Lett. 91, 096102 (2003).

${ }^{5}$ J.-L. Lin, D. Y. Petrovykh, J. Viernow, F. K. Men, D. J. Seo, and F. J. Himpsel, J. Appl. Phys. 84, 255 (1998); J. Viernow, J.-L. Lin, D. Y. Petrovykh, F. M. Leibsle, F. K. Men, and F. J. Himpsel, Appl. Phys. Lett. 72, 948 (1998).

${ }^{6}$ H. Omi and T. Ogino, J. Vac. Sci. Technol. A 17, 1610 (1999); A. Kirakosian, R. Bennewitz, J. N. Crain, Th. Fauster, J.-L. Lin, D. Y. Petrovykh, and F. J. Himpsel, Appl. Phys. Lett. 79, 1608 (2001).

${ }^{7}$ S. Yoshida, T. Sekiguchi, and K. M. Itoh, Appl. Phys. Lett. 87, 031903 (2005).

${ }^{8}$ J. L. McChesney, A. Kirakosian, R. Bennewitz, J. N. Crain, J-L. Lin, and F. J. Himpsel, Nanotechnology 13, 545 (2002).

${ }^{9}$ T. Sekiguchi, S. Yoshida, and K. M. Itoh, Phys. Rev. Lett. 95, 106101 (2005); K. N. Romanyuk, S. A. Teys, and B. Z. Olshanetsky, Phys. Solid State 48, 1820 (2006); T. Sekiguchi, S. Yoshida, K. M. Itoh, J. Mysliveček, and B. Voigtländer, Appl. Phys. Lett. 90, 013108 (2007).

${ }^{10}$ M. Nakaya, T. Nakayama, Yu. Kuwahara, and M. Aono, Surf. Sci.
600, 2810 (2006).

${ }^{11}$ M. Copel, M. C. Reuter, E. Kaxiras, and R. M. Tromp, Phys. Rev. Lett. 63, 632 (1989); M. Horn-von Hoegen, F. K. LeGoues, M. Copel, M. C. Reuter, and R. M. Tromp, ibid. 67, 1130 (1991); M. Copel, M. C. Reuter, M. Horn von Hoegen, and R. M. Tromp, Phys. Rev. B 42, 11682 (1990); G. Meyer, B. Voigtländer, and N. M. Amer, Surf. Sci. Lett. 274, L541 (1992).

${ }^{12}$ B. Voigtländer, M. Kawamamura, N. Paul, and V. Cherepanov, Thin Solid Films 464, 185 (2004).

${ }^{13}$ N. Paul, H. Asaoka, J. Mysliveček, and B. Voigtländer, Phys. Rev. B 69, 193402 (2004); N. Paul, H. Asaoka, and B. Voigtländer, Surf. Sci. 564, 187 (2004).

${ }^{14}$ K. J. Wan, T. Guo, W. K. Ford, and J. C. Hermanson, Phys. Rev. B 44, 3471 (1991).

${ }^{15}$ R. Z. Bakhtizin, Ch. Park, T. Hashizume, and T. Sakurai, J. Vac. Sci. Technol. B 12, L290 (1990).

${ }^{16}$ T. Schmidt, J. Falta, and G. Materlik, Appl. Phys. Lett. 74, 1391 (1999).

${ }^{17}$ S. Hasegawa, R. G. Ryland, and E. D. Williams, Appl. Phys. Lett. 65, 2609 (1994).

${ }^{18}$ T. Schmidt, J. Falta, and G. Materlik, Appl. Surf. Sci. 166, 399 (2000).

${ }^{19}$ N. Paul and B. Voigtländer, Surf. Sci. 551, 80 (2004).

${ }^{20}$ V. Cherepanov, S. Filimonov, J. Mysliveček, and B. Voigtländer, Phys. Rev. B 70, 085401 (2004).

${ }^{21}$ I. Štich, M. C. Payne, R. D. King-Smith, J. S. Lin, and L. J. Clarke, Phys. Rev. Lett. 68, 1351 (1992).

${ }^{22}$ M. Fonin, J. M. Choi, U. May, J. O. Hauch, U. Rüdiger, and G. Güntherodt, Surf. Sci. 511, 312 (2002). 